\title{
Out-of-plane motion estimation based on a Rician-Inverse Gaussian model of RF ultrasound signals: speckle tracking without fully developed speckle
}

\author{
N. Afsham, M. Najafi, P. Abolmaesumi, R. Rohling \\ Dept. of Electrical and Computer Engineering, UBC, Vancouver, BC, Canada
}

\begin{abstract}
Fully developed speckle has been used previously to estimate the out-of-plane motion of ultrasound images. However, in real tissue the rarity of such patterns and the presence of coherency diminish both the precision and the accuracy of the out-of-plane motion estimation. In this paper, for the first time, we propose a simple mathematical derivation for out-ofplane motion estimation in which the coherent and non-coherent parts of the RF echo signal are separated. This method is based on the Rician-Inverse Gaussian stochastic model of the speckle formation process, which can be considered as a generalized form of the K-distribution with richer parameterization. The flexibility of the proposed method allows considering any patch of the RF echo signal for the purpose of displacement estimation. The experimental results on real tissue demonstrate the potential of the proposed method for accurate out-of-plane estimation. The underestimation of motion in ex vivo bovine tissue at $1 \mathrm{~mm}$ displacement is reduced to $15.5 \%$ compared to $37 \%$ for a base-line method.
\end{abstract}

Keywords: Sensorless freehand ultrasound, speckle, fully developed speckle, out-of-plane motion

\section{INTRODUCTION}

The deviation of a coherent field phase front from its original form, after confronting a random medium, results in a granular noise-like pattern in ultrasound images referred to as speckle ${ }^{1}$. Speckle is an informative signal rather than being random noise and it can be used to reveal information about the imaging system or the random medium ${ }^{2}$. On the other hand, the speckle formation process can be considered as the summation of small phase changes of the incident coherent signal produced by randomly distributed scatterers ${ }^{3}$. Such a process intrinsically suggests it can be modeled as a stochastic process. By modeling the speckle formation process, it is possible to obtain information about the echo signal amplitude and intensity distributions.

Sensorless freehand ultrasound aims to eliminate the need of a position sensor mounted on the transducer with speckle tracking. The main challenge is the out-of-plane motion estimation. Three main categories have been introduced in the literature for the purpose of speckle tracking: regression-based, correlation-based, and learning-based methods.

The regression-based method ${ }^{4}$ determines the best affine linear estimation of a circularly Gaussian distributed Radio Frequency (RF) signal correspondent to a Fully Developed Speckle (FDS) pattern and is closely related to the correlation of speckle patches. The majority of the remaining work on ultrasound speckle tracking is correlation-based. The second order statistics and low-order moments of the envelope ultrasound echo signals has been used to estimate the out-of-plane motion for more than two decades ${ }^{5}$. Many of the proposed correlation-based methods focus on the improvement of FDS detection by introducing different FDS detection methods, such as optimal low-order moments ${ }^{6}$, novel meshing ${ }^{7}$, and the use of the K-S test as a non-parametric goodness of fit ${ }^{8}$. Other efforts intend to increase the motion estimation accuracy by adapting the correlation curve and compensating the loss of coherency by using additional information such as correlation in the axial and lateral directions ${ }^{8}$, beam steering ${ }^{9}$, developing a heuristic method to consider the coherent part of the image ${ }^{10}$, using Maximum Likelihood Estimators (MLE) for motion estimation ${ }^{11}$, or incorporating the information of several noisy measurements in a probabilistic framework ${ }^{12}$. Recently a new learning based method of out-of-plane motion estimation on imagery of real tissue has been introduced ${ }^{13}$. They adapt the scale factor of the nominal correlation curve based on training data.

In this paper, we have developed a novel correlation-based method that incorporates coherency in the derivation of correlation function and gives a general form of previously proposed correlation-based methods. For any patch of the image, the parameters of the Rician-Inverse Gaussian (RiIG) model are estimated from the RF signal, and from these

Medical Imaging 2012: Ultrasonic Imaging, Tomography, and Therapy, edited by Johan G. Bosch,

Marvin M. Doyley, Proc. of SPIE Vol. 8320, 832017 - () 2012 SPIE

CCC code: $1605-7422 / 12 / \$ 18 \cdot$ doi: $10.1117 / 12.911710$

Proc. of SPIE Vol. $8320832017-1$ 
parameters, the correlation coefficient is computed. Since we propose an analytical closed-form formula for the correlation, it is possible to estimate the out-of-plane motion. The remaining parameter needed in this formulation is the elevation width of the ultrasound Point Spread Function (PSF) in its elevation direction which can be found from a prior calibration process.

\section{METHODS}

\subsection{Speckle formation process}

As mentioned, the speckle formation process can be modeled as a stochastic process. It can be stated as the summation of $N$ complex phasors as below ${ }^{2}$ :

$$
A=\sum_{i=1}^{N}\left|a_{i}\right| e^{j \emptyset_{i}}=X_{r}+j Y_{r} .
$$

$A$ is the complex RF signal and $N$ is the number of scatterers in a resolution cell. The number of scatterers in a resolution cell for a randomly distributed medium follows a Poission process ${ }^{14}$. If the number of scatterers is large enough, the amplitude of the radio-frequency signal, $R=\sqrt{X_{r}^{2}+Y_{r}^{2}}$, has a Rayleigh distribution. In this case in-phase (I) and quadrature $(\mathrm{Q})$ components of the RF signal, $X_{r}$ and $Y_{r}$, are zero-mean Gaussian distributed. The Rayleigh distribution is correspondent to FDS.

If the variances of I and Q components of the RF signal are $\Gamma$ distributed, the RF echo signal is $\mathrm{K}$ distributed ${ }^{14}$. In a general form, $X_{r}$ and $Y_{r}$ are compound random variables as follows:

$$
\begin{aligned}
& X_{r}=\mu_{x}+\beta_{x} Z+\sqrt{Z} N_{x}, \\
& Y_{r}=\mu_{y}+\beta_{y} Z+\sqrt{Z} N_{y} .
\end{aligned}
$$

$N_{x}$ and $N_{y}$ are normally distributed with a covariance matrix of identity.

If $\mathrm{Z}$ is $\Gamma$ distributed, with $\mu_{x}=\mu_{y}=0, R$ has a Rician distribution. If $\beta_{x}=\beta_{y}=0, R$ becomes homodyned-K distributed $^{15}$. There is no explicit analytical expression for homodyned-K Probability Density Function (PDF) and its second order statistics; hence, it is more difficult to use for the application of the out-of-plane motion estimation.

Considering the Inverse Gaussian (IG) distribution for $Z$ and zero $\mu_{x}$ and $\mu_{y}$, the resulting distribution is the RiIG ${ }^{16}$. The physical interpretation of RiIG is that the complex RF signal is a combination of two independent Brownian motion with drifts $\beta_{x}$ and $\beta_{y}$ and the Inverse Gaussian (IG) first passage time. The drift part models the presence of coherency in the echo signal. In this statistical process, $Z$ models the speckle-free part of the signal and $N_{x}, N_{y}$ correspond to the FDS part. A previous study on RiIG shows that it outperforms K, and Nakagami distributions in modeling ultrasound echo signal ${ }^{14}$. Moreover, it has some interesting characteristics that make it suitable for the application of out-of-plane motion estimation. It is possible to estimate the parameters of RiIG distribution fairly well, even from a few samples ${ }^{14}$. Moreover, its posterior distribution formula is available in a closed form that makes it possible to estimate the coherent part, $Z$, in a Maximum A-Posterior (MAP) manner ${ }^{16}$. Finally, the model allows for separation of the coherent and noncoherent parts of the correlation function, as will be described next.

\subsection{Motion estimation based on second order statistics}

For a linear rectangular array, the point spread function (PSF) of the returned echo amplitude can be simplified as ${ }^{17}$ :

$$
g(x, y, z)=g(x, z)_{\text {in_plane }} g(y)_{\text {out_of_plane }}=K g(x, z) \cdot \operatorname{sinc}^{2}\left(\frac{y}{\sigma_{y}}\right)
$$

where y indicates the out-of-plane or elevation direction. $\operatorname{sinc}(y)$ equals $\frac{\sin (\pi y)}{\pi y}$ and $\sigma_{y}=\frac{\lambda_{0} z_{0}}{D_{y}} \cdot D_{y}$ refers to transducer dimension in the elevation direction. $z_{0}$ is the distance along the beam axis and $\lambda_{0}$ is the ultrasound wavelength in its central frequency. $K$ represents a constant factor. 
For a random process $A$ and spatial point spread function of an imaging system $g(x, y, z)$, if the displacement is only in elevation direction, based on the linear system theory, the autocorrelation function of the backscattered complex process can be written as ${ }^{2}$ :

$$
R(\Delta y)=R_{A}(\Delta y) * g(-\Delta y) * g^{*}(\Delta y),
$$

where $*$ indicates the convolution operator and $g *$ is the complex conjugate of $g$.

In the case of a rectangular array $g(y)$ can be approximated by $\operatorname{sinc}^{2}$ function and it gives:

$$
R(\Delta y)=K^{2} R_{A}(\Delta y) * \operatorname{sinc}^{2}\left(\frac{\Delta y}{\sigma_{y}}\right) * \operatorname{sinc}^{2}\left(\frac{\Delta y}{\sigma_{y}}\right) .
$$

The very simple approximation of the convolution of two $\operatorname{sinc}^{2}$ functions is a Gaussian one. However, such an approximation may be one of the sources of the out-of-plane motion underestimation based of Gaussian correlation curves reported in previous papers. Since the convolution of two $s i n c^{2}$ functions can be determined by term-wise Fourier transform ${ }^{2}$, it gives:

$$
R(\Delta y)=R_{A}(\Delta y) * \frac{K^{2}}{(\pi \Delta y)^{2}}\left(1-\operatorname{sinc}\left(\frac{2 \Delta y}{\sigma_{y}}\right)\right) .
$$

Under Rayleigh conditions, where the microstructure of the imaging sample is uncorrelated as a result of randomly scattered fine particles in the imaging sample, and due to the averaging over uniformly distributed phases we have ${ }^{2}$ :

$$
R_{A_{\text {Rayleigh }}}(\Delta y)=2 \sigma_{d}^{2} \delta(\Delta y) .
$$

$\sigma_{d}^{2}$ is the variance of the diffuse part. In this case, the autocorrelation of the output process only reveals the information of imaging PSF $(g(y))$ rather than the information of medium and it can be used to estimate the out-of-plane motion $(\Delta y)$.

In the case of RiIG process, autocorrelation function can be written as:

$$
\begin{aligned}
R_{A}(\Delta y)=< & \left(\beta_{r} Z_{1}+\sqrt{Z_{1}} N_{x 1}+j\left(\beta_{i} Z_{1}+\sqrt{Z_{1}} N_{y 1}\right)\right)\left(\beta_{r} Z_{2}+\sqrt{Z_{2}} N_{x 2}+j\left(\beta_{i} Z_{2}+\sqrt{Z_{2}} N_{y 2}\right)\right)^{*}> \\
= & <\sqrt{Z_{1} Z_{2}}><N_{x 1} N_{x 2}>+<\sqrt{Z_{1} Z_{2}}><N_{y 1} N_{y 2}>+\beta^{2}<Z_{1} Z_{2}>.
\end{aligned}
$$

We assumed that $\beta_{r}$ and $\beta_{i}$ are the same for the closely positioned frames and $\beta=\sqrt{\beta_{r}^{2}+\beta_{i}^{2}}$. 1 and 2 subscripts represent the process at the positions $y_{1}$ and $y_{2}$ respectively.

Considering $Z_{1}$ and $Z_{2}$ fully correlated for two adjacent frames, without loss of generalization, it can be assumed $Z_{2}=k Z_{1}$ or $Z_{1}=k Z_{2}$ such that $0 \leq k \leq 1$. Considering the fact that $Z$ models the variance of the first passage time of the process, it is plausible to suppose that $Z$ is independent of the normally distributed part. Under these circumstances:

$$
R_{A}(\Delta y)=\sqrt{k}<N_{x 1} N_{x 2}><Z>+\sqrt{k}<N_{y 1} N_{y 2}><Z>+k \beta^{2}<Z^{2}>\text {. }
$$

Since the Rayleigh condition goes for the normal part of RilG distribution and the variances of these normal distributions are unit, we can replace $<N_{x 1} N_{x 2}>$ by $\delta(\Delta y)$ (see ${ }^{2}$ for more details on the derivation of Rayleigh and $\mathrm{K}$ correlation functions).

$$
R_{A}(\Delta y)=2 \sqrt{k}<Z>\delta(\Delta y)+k \beta^{2}<Z^{2}>.
$$

In this new correlation function, the variance of the diffuse part is replaced by $2 \sqrt{k}<Z>$ (compare with Eq. 7) and $k \beta^{2}<Z^{2}>$ represents the effect of coherent part.

Substituting the moment of $Z$ from ${ }^{14}$ in Eq. 9 and apply the result in Eq. 5, we may write:

$$
R(\Delta y)=R_{A}(\Delta y) * \frac{K^{2}}{(\pi \Delta y)^{2}}\left(1-\operatorname{sinc}\left(\frac{2 \Delta y}{\sigma_{y}}\right)\right) .
$$

After some simple arithmetic: 


$$
R(\Delta y)=K^{2} k \beta^{2}\left(\frac{\delta}{\gamma}\right)^{2}\left(1+\frac{1}{\delta \gamma}\right) \sigma_{y}^{2}+K^{2}\left(\frac{\delta}{\gamma}\right) \frac{2 \sqrt{k}}{(\pi \Delta y)^{2}}\left(1-\operatorname{sinc}\left(\frac{2 \Delta y}{\sigma_{y}}\right)\right)=R_{c}+R_{r}(\Delta y) .
$$

$R_{c}$ is the coherent part of the correlation and $R_{r}$ is the Rayleigh part which out-of-plane displacement can be estimated from. To eliminate the scaling factor $\mathrm{K}$, it is feasible to use correlation coefficient instead:

$$
\rho=\frac{C(\Delta y)}{C(0)}=\frac{R(\Delta y)-\mu_{A}\left(y_{1}\right) * g(y) \times \mu_{A}\left(y_{2}\right) * g(y)}{\sigma_{A}^{2}},
$$

where $\mu_{A}(y)$ is mean of the process at position $y$ and $\sigma_{A}^{2}$ is the variance of the process. Assuming $A$ is a stationary process to its second order, and using the explicit terms for RiIG moments ${ }^{14}$, from Eq.12 and Eq. 13 the correlation coefficient is given by:

$$
\rho=\frac{k \beta^{2} \sigma_{y}^{2}+2 \gamma^{2} \sqrt{k}\left(\frac{1-\operatorname{sinc}\left(\frac{2 \Delta y}{\sigma_{y}}\right)}{(\pi \Delta y)^{2}}\right)}{\beta^{2} \sigma_{y}^{2}+\frac{4}{3 \sigma_{y}^{2}} \gamma^{2}} .
$$

The maximum value of function $\left(\frac{1-\operatorname{sinc}\left(\frac{2 \Delta y}{\sigma_{y}}\right)}{(\pi \Delta y)^{2}}\right)$ equals $\frac{2}{3 \sigma_{y}^{2}}$ and $0 \leq k \leq 1$, so $\rho$ is less than 1 for all the values of $\Delta y$, as expected.

$\rho$ is directly calculated from the ultrasound RF signal. All the parameters in the right side of Eq. 14, including $k, \beta$, and $\gamma$, can be estimated from data based on Expectation Maximization algorithm (EM) explained in ${ }^{14},{ }^{16} . \sigma_{y}$ is the variance of the imaging system PSF at different depths, which can be known from the system manufacturer or can be calibrated from a speckle phantom. The second approach has been followed in this work.

The mathematical expression for MAP estimation of the coherent part $(Z)$ of the RF signal can be written as:

$$
\hat{z}=\arg \max p_{Z \mid R}(z \mid r) .
$$

Since the posterior distribution of $Z, p_{Z \mid R}(z \mid r)$, is given in a closed form ${ }^{16}, \hat{z}$ is explicitly determined. For the details and discussion on the parameter estimation of RiIG model see ${ }^{14}$.

\section{EXPERIMENTAL SETUP}

The image acquisition system consists of a $10 \mathrm{MHz}$ 2D linear probe (SonixGPS, Ultrasonix Inc., Richmond, BC, Canada). The elevation movement of the phantom is created by means of a linear motor stage (T-LSR150B, Zaber Technologies Inc., Vancouver, BC, Canada) with equal steps of $0.0635 \mathrm{~mm}$. The ultrasound probe is fixed during the experiments and the phantom is placed on top of the motor stage. We considered the frames to be parallel with no inplane motion.

Firstly in a calibration process, a phantom with a large number of randomly distributed scatterers is scanned. At four different axial depths of the image $(0.57,0.95,1.33,1.75 \mathrm{~cm})$ a patch of $100 \mathrm{RF}$ sample $\times 25$ pixel is considered. The PSF elevation width of the ultrasound transducer $\left(\sigma_{y}\right)$ is estimated at each depth from the selected patch to minimize the

difference of true displacement and estimated ones from a sequence of 40 frames with equal steps of $0.0635 \mathrm{~mm}$ displacements. Then, the same experiment is performed on real tissue. At each depth, similar to the depths selected in the calibration phase, a random window of $100 R F$ sample $\times 25$ pixel is selected. Here for the sake of simplicity we used non overlapping windows, but it is possible to apply this method on any arbitrary window at the selected depth. The parameters of the RiIG model are estimated as explained previously. Since all of the parameters used in Eq. 14 are now determined, the out-of-plane motion is computable. Note that the value of $\sigma_{v}$ for each depth is different and comes from the calibration process. 


\section{RESULTS}

Fig. 1 shows samples of estimated distributions of $R$ and $Z$ from a phantom image. RiIG is a distribution with three parameters: $\beta, \gamma$ and $\delta$. The EM algorithm has been used to estimate these parameters from $20 \times 20,50 \times 50$, and 100 $R F$ sample $\times 25$ pixel patches at the same center position. Figure 1 summarizes the result. The PDF of the RF amplitude samples of the phantom is compared to the one which is generated from the closed-form formula and the estimated parameters. $Z$ is determined from MAP estimation and it is compared to the PDF resulted from closed-form formulation of IG model. It is observed that estimated PDFs follow the real ones fairly well, even for small numbers of samples. Fig. 1 indicates that as the number of samples increases, the closer estimation to the real data is acquired.

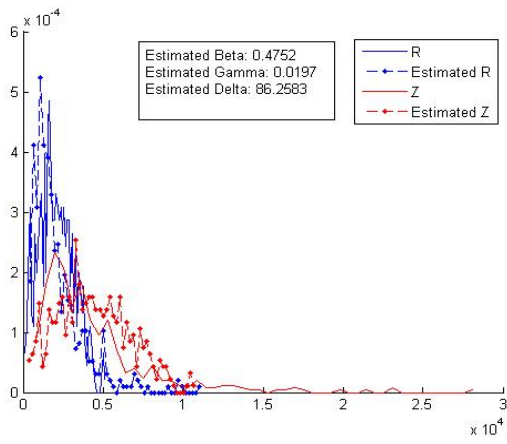

(a)

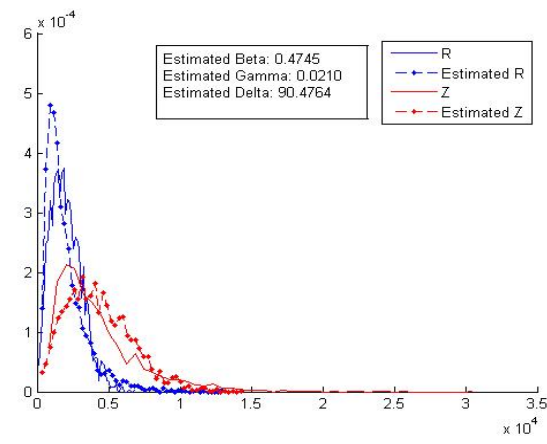

(b)

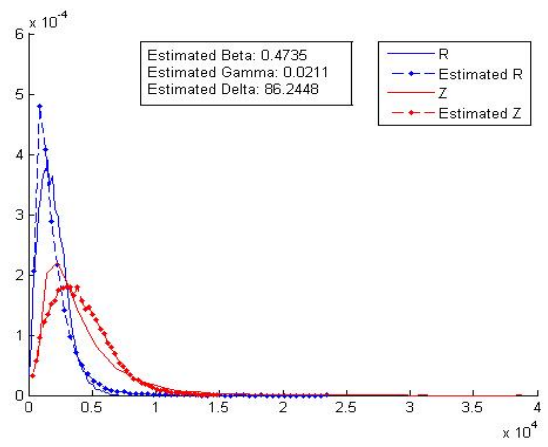

(c)

Fig. 1 Estimated PDF in comparison with real PDF from (a) $20 \times 20$ (b) $50 \times 50$ and (C) $100 \times 100$ patches

To evaluate the proposed method, the base-line method of low-order moments for FDS detection ${ }^{18}$ has been implemented and a Gaussian curve has been fitted on the correlation curve of the phantom data. As Fig. 2(a) shows, FDS patches are rare within the real tissue and no FDS patch is found at the third depth. Underestimation of displacement in the proposed method occurs at greater depths in comparison with the FDS-based method. The average underestimation of our implementation of a base-line method at $1 \mathrm{~mm}$ displacement is $37 \%$ compared to $15.5 \%$ with the proposed method.

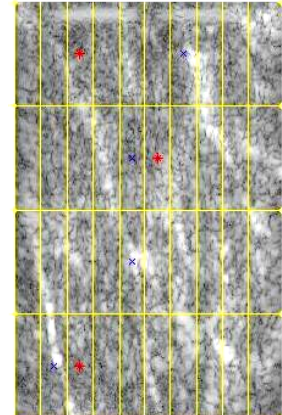

(a)

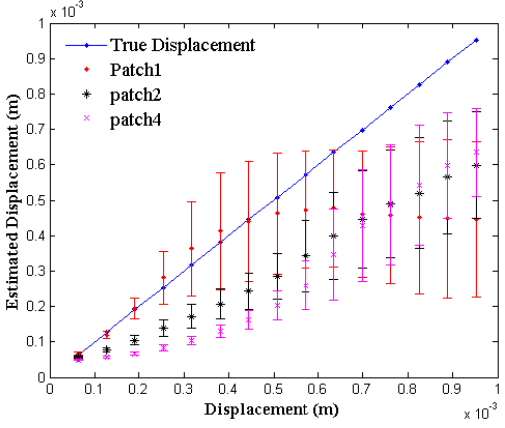

(b)

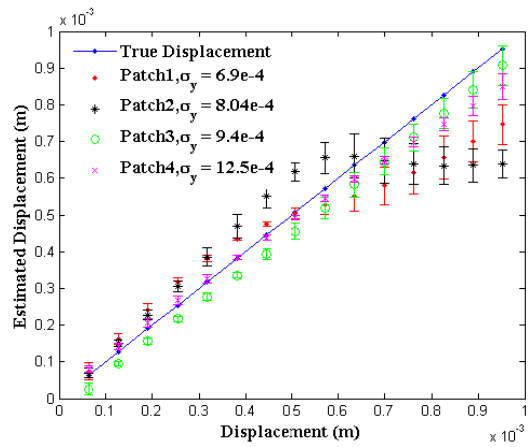

(c)

Fig.2 (a) Bovine ultrasound image (red star indicates FDS patch and blue $\mathrm{x}$ shows randomly selected patch at each depth) (b) Displacement estimation using base-line FDS method for three patches (c) Displacement estimation using the proposed method

\section{DISCUSSION AND CONCLUSION}

One of the main concerning issues in FDS-based freehand ultrasound is the rarity of FDS patches in real tissue. The presence of coherency causes underestimation of out-of-plane motion as the displacement increases. We proposed a novel correlation-based method of out-of-plane motion estimation for non-FDS patches. The method is based on the RiIG model of speckle formation process which is a general representation for ultrasound echo intensity. This model provides the possibility of dividing the correlation of two patches into two terms. The one that shows the coherency of 
the patches and the one that comes from the randomly located scatterers. In this frame work it is possible to use almost all patches of the images regardless of being FDS and take advantage of plenty estimations and make the final out-ofplane motion estimation more robust.

Here we just evaluated the out-of-plane motion estimation for the frames with fixed elevation displacement. It is possible to determine the complete out-of-plane transform between two frames based on the elevation distance of tree different corresponding points on the frames ${ }^{10}$.

Experiments on a tissue sample suggest that considering the coherent part significantly improves both the precision and the accuracy of the out-of-plane motion estimation. Our results indicate by choosing a suitable frame distance (around $0.6 \mathrm{~mm}$ in this experiment) the underestimation over a long distance can be considerably reduced. More experiments are needed to demonstrate ability on tracking in vivo.

\section{ACKNOWLEGMENTS}

This work is supported by the Natural Sciences and Engineering Research Council of Canada (NSERC) and Canadian Institutes of Health Research (CIHR).

\section{REFERENCES}

[1] Abbott, J.G., and Thurstone, F.L., “Acoustic speckle: Theory and experimental analysis,” Ultrasonic Imaging 1(4), 303-324 (1979).

[2] Wagner, R.F., Smith, S.W., Sandrik, J.M., and Lopez, H., "Statistics of speckle in ultrasound B-scans," IEEE transactions on sonics and ultrasonics 30(3), 156-163 (1983).

[3] Middleton, D., [An Introduction to Statistical Communication Theory: An IEEE Press Classic Reissue], 1st ed., Wiley-IEEE Press (1996).

[4] Prager, R.W., Gee, A.H., Treece, G.M., Cash, C.J.C., and Berman, L.H., "Sensorless freehand 3-D ultrasound using regression of the echo intensity," Ultrasound in Medicine \& Biology 29(3), 437-446 (2003).

[5] Chen, J.-F., Fowlkes, J.B., Carson, P.L., and Rubin, J.M., "Determination of scan-plane motion using speckle decorrelation: Theoretical considerations and initial test," International Journal of Imaging Systems and Technology 8(1), 38-44 (1997).

[6] Martí, R., Martí, J., Freixenet, J., Zwiggelaar, R., Vilanova, J.C., and Barceló, J., “Optimally discriminant moments for speckle detection in real B-scan images," Ultrasonics 48(3), 169-181 (2008).

[7] Rivaz, H., Boctor, E., and Fichtinger, G., "A robust meshing and calibration approach for sensorless freehand 3D ultrasound," in Medical Imaging 2007: Ultrasonic Imaging and Signal Processing 6513, 651318-8 (2007).

[8] Hassenpflug, P., Prager, R.W., Treece, G.M., and Gee, A.H., "Speckle classification for sensorless freehand 3-D ultrasound," Ultrasound in Medicine \& Biology 31(11), 1499-1508 (2005).

[9] Rivaz, H., Zellars, R., Hager, G., Fichtinger, G., and Boctor, E., "Beam Steering Approach for Speckle Characterization and Out-of-Plane Motion Estimation in Real Tissue," in IEEE Ultrasonics Symposium, 2007, 781-784 (2007).

[10] Gee, A.H., James Housden, R., Hassenpflug, P., Treece, G.M., and Prager, R.W., "Sensorless freehand 3D ultrasound in real tissue: Speckle decorrelation without fully developed speckle," Medical Image Analysis 10(2), 137-149 (2006).

[11] Rivaz, H., Kang, H.J., Stolka, P.J., Zellars, R., Wacker, F., Hager, G., and Boctor, E., "Novel reconstruction and feature exploitation techniques for sensorless freehand 3D ultrasound," in Medical Imaging 2010 SPIE Proceeding, 76291-76299 (2010).

[12] Laporte, C., and Arbel, T., "Combinatorial and probabilistic fusion of noisy correlation measurements for untracked freehand 3-D ultrasound," IEEE Transactions on Medical Imaging 27(7), 984-994 (2008).

[13] Laporte, C., and Arbel, T., "Learning to estimate out-of-plane motion in ultrasound imagery of real tissue," Medical Image Analysis 15(2), 202-213 (2011).

[14] Eltoft, T., "Modeling the amplitude statistics of ultrasonic images," IEEE Transactions on Medical Imaging 25(2), 229-240 (2006).

[15] Eltoft, T., "A new approach to modeling signal amplitude statistics by the K distributions," in Proceedings of the 7th Nordic, 62-65 (2006). 
[16] Eltoft, T., "The Rician inverse Gaussian distribution: a new model for non-Rayleigh signal amplitude statistics," IEEE Transactions on Image Processing: A Publication of the IEEE Signal Processing Society 14(11), 17221735 (2005).

[17] Chen, J.-F., Fowlkes, J.B., Carson, P.L., and Rubin, J.M., "Determination of scan-plane motion using speckle decorrelation: Theoretical considerations and initial test," International Journal of Imaging Systems and Technology 8(1), 38-44 (1997).

[18] Prager, R.W., Gee, A.H., Treece, G.M., and Berman, L.H., "Analysis of speckle in ultrasound images using fractional order statistics and the homodyned k-distribution," Ultrasonics 40(1), 133-137 (2002). 